\title{
Is bicarbonate in Photosystem II the equivalent of the glutamate ligand to the iron atom in bacterial reaction centers?
}

\author{
X. Wang ", J. Cao ", P. Maróti ", H.U. Stilz ", U. Finkele ', C. Lauterwasse ", \\ W. Zinth " ${ }^{c}$ D. Oesterheit ", Govindjee " and C.A. Wraight " \\ "Unicersity of Illinois, Urbana, IL 61801 (USA). "Max.Plank-Institu fü Biochemic, 8033. Marninsried (Germany) \\ and 'Physik Department. Technische Unil ersitür. D-80(k) Mäncho'n (Germamy)
}

(Received 15 August 1991)

(Revised manuscript received of Decemher [99])

Key words: Bicarhonate effect; Electron acceptor complex; Electron transfer; Photosynthesis; Quinone: Reaclion center: Site-directed mulagenesis: ( $R b$. sphateroides)

Photosystem II of oxygen-evolving organisms cxhibits a bicarbonatc-reversible formate effect on electron transfer between the primary and secondary acceptor quinones. $Q_{A}$ and $Q_{B}$. This effect is absent in the otherwise similar electron acceptor complex of purple bacteria, e.g., Rhodobacter sphaeroides. This distinction has led to the suggestion that the iron atom of the acceptor quinone complex in PS II might lack the fifth and sixth ligands provided in the bacterial reaction eenter (RC) by a glutamate residue at position 234 of the M-subunit in Rb. sphaeroides RCs (M232 in Rps. tiritis). By site-directed mutagenesis we have altered Glu ${ }^{M 234}$ in RCs from $R b$. sphaeroides, replacing it with valine. glutamine and glycinc to form mutants $M 234 E V$. M234EQ and M234EG, respectively. These mutants grew competently under phototrophic conditions and were tested for the formate-bicarbonate effect. In chromatophores there were no detectable differences between wild type (Wt) and mutant M234EV witls respect to cytochrome $\$$-561 reduction following a flash, and no effect of bicarbonate depletion (by incubation with formate). In isolated RCs, several electron transfer activities were essentially unchanged in $W \mathrm{t}$ and $\mathrm{M} 234 \mathrm{EV}, \mathrm{M} 234 \mathrm{EQ}$ and $\mathrm{M} 234 \mathrm{EG}$ mutants, and no formate-bicarbonate effect was observed on: (a) the fast or slow phases of recovery of the oxidized primary donor $\left(P^{+}\right)$in the absence of exogenous donor, i.c., the recombination of $P^{+} Q_{A}$ or $P^{+} Q_{B}$. respectively; (b) the kinetics of clectron transfer from $Q_{A}^{-}$to $Q_{B}$; or (c) the flash dependent osciliations of semiquinone formation in the presence of donor to $\mathrm{P}^{+}\left(\mathrm{O}_{\mathrm{B}}\right.$ turnover $)$. The absence of a formate-bicarbonate effect in these mutants suggests that Glu ${ }^{\mathrm{M} 23}$ is not responsible for the absence of the formatc-bicarbonate effect in Wt bacterial RCs, or at least that other factors must be taken into account. The mutant RCs were also examined for the fast primary electron transfer along the active (A-)branch of the pigment chairi. leading to reduction of $Q_{A}$. The kinctics were resolved to reveal the reduction of the monomer bacteriochlorophyll $(;=3.5 \mathrm{ps})$. followed by reduction of the bacteriopheophytin $(\tau=0.9 \mathrm{ps})$. Both steps were essentially unaltered from the wild type. However. the ratc of reduction of $Q_{A}$ was slowed by a factor of $2(\tau=410 \pm 30$ and $47 \pm 30 \mathrm{ps}$ for M234EQ and M234EV. respectively, compared to $220 \mathrm{ps}$ in the wild type). EPR studies of the isolated RCs showed a characteristic $g=1.82$ signal for the $Q_{\mathrm{A}}$ semiquinone compled to the iron atom, which was indistinguishable from the wild type. It is concluded that Glu ${ }^{\text {M234 }}$ is not essential to the normal functioning of the acceptor quinone complex in bacterial RCs and that the role of bicarbonate in PS II is distinct from the role of this residue in baclerial RCs.

\section{Introduction}

Electron fiow through the quinone electron acceptors of Photosystem II (PS II) in oxygen-evolving organisms is inhibited by several monovalent anions, of which formate is onc of the most active [1]. Addition of bicarbonate reverses this inhibition producing a large stimulation of electron transfer from $Q_{A}$, the primary

Correspondence: C. Wraight, Department of Plant Biology, 162 PABL. 1201 West Gregory Drive. Urbana IL 01801, USA. quinone, to $Q_{B}$, the secondary quinone acceptor of PS II [2]. It is considered that bicarbonate rather than $\mathrm{CO}_{2}$ is the species required for the stimulation [3]. The antagonistic action of formate and bicarbonate on electron transfer is termed the bicarbonate effect and is considered to be a manifestation of a genuine bicarbonate requirement. However, the effect is difficult to demonstrate directly by depletion in the absence of formate [4]. Bicarbonate depletion results in a small (2-3-fold) decrease in the rate of the first electron transfer from $Q_{A}^{-}$to $Q_{B}$, a more marked inhibition of the second electron transfer from $Q_{A}^{-}$to $Q_{B}^{-}$and a 
significant inhibition of the release of $\mathrm{Q}_{13} \mathrm{H}_{2}$ from the raction center.

The site of the bicarbonate effect has been shown to be the acceptor quinone complex of PS 1I [4]. However. in purple bacteria. which possess a functionally very similar acceptor quinone complex to that of PS II, no bicarbonate effect could be demonstrated. either in isolated reaction centers (RCs) or in chromatophores of Rhodobacter sphaeroides [5]. The X-ray crystallographic structures of RCs from Rhodopsendomonas tiridis [6] and Rb. sphaeroides [7.8] are known. and the acceptor quinone complex is seen to include a nonhacm iron coordinated by four histidines and by a bidentate ligand interaction with the carboxylate group of a glutamate residue (M234 in the Rb. sphacroides sequence). Comparison of the amino-acid sequences of RC proteins from PS II indicates that the subunit structure of PS II and purple bacterial RCs are roughly homologous, with D1 and D2 of PS II equivalent to the $L$ and $M$ subunits of bacterial RC. Amino-acid scquence differences in the polypeptides of PS II and hacterial RCs also led to the suggestion that the different responses to bicathonate might $b$ ? we to the provision of the fifth and sixth ligands to the iron atom by Glu ${ }^{1234}$ in bacteria and the absence of this linkage in PS II; in PS II this role might then be filled by bicarbonate [9]. Direct interaction of some exogenous ligands with the iron atom of PS II is supported by EPR and Mössbauer studies, and modulation of these effects by $\mathrm{HCO}_{3}^{-}$was interpreted as indicating that bicarbonate could be the fifth, and possibly the sixih. ligand to the non-haem iron of the acceptor quinone complex [10]. Some studies have implicated more than one site of action for $\mathrm{HCO}_{3}^{-}$and a salt bridge function has also been postulated, as partner to an arginine [4]. We attempted to test the first proposal by replacing Glu ${ }^{\text {M234 }}$ with valine, glutamine and glycine. All three mutants behaved similarly to the wild type and none exhibited a bicarbonate effect. implying that the absence of Glu ${ }^{\mathrm{M} 234}$ is not a sufficient requirement for this effect.

A preliminary report of this work was presented at the 35th Annual Mecting of the Biophysical Society. February. 1991, San Francisco. USA (Biophys. J. 59. 146a.)

\section{Materials and Methods}

Site-directed mutagenesis. Site-directed mutations of $R h$. sphaeroides DNA were generated using two similar vector and expression systems described more fully eisewhere [11.12]. All constructions were based on the $R b$. sphacroides reaction center operon ( puf), originally isolated as a $13 \mathrm{~kb}$ Bam Hl fragment in pBR322 (pJW1; see Ref. 13). Deletion strains for expression of mitant genes from a plasmid were obtained through
Jouble recombination with a suicide plasmid (pSUP202; see Ref. 14) containing the flanking DNA regions of the $R b$. spinacroides puf operon, with the operon, itself, replaced by a kanamycin resistance cassette $(\mathrm{Km})$. The vectors for expression of mutant genes were based on pRK404, using either a $5.3 \mathrm{~kb}$ Bam HI/Hindll fragment [11] containing the $R b$. sphaeroides puf operon, or a $4.5 \mathrm{~kb} P_{S t} I$ fragment functioning under the $\mathrm{Tc}^{r}$ promoter of pRK4(14 [12]. Site directed mutagenesis was performed by standard techniques $[15,16]$ either on the entire expression fragment or on smaller subclones. After sequencing to select and confirm the mutations, tice expression fragments were cloned into pRK404 (car ying the tetracycline resistance marker, $\mathrm{Tc}^{r}$ ) and transferred into a deletion strain of $R b$. sphaeroides by diparental conjugation with the mobilizing strain of $E$. coli, S17-1 [14,17]. In one case, for the mutation Glu $^{\mathrm{M} 274} \rightarrow \mathrm{Val}$, the mutated gene was also incorporated into the genome by double recombination with the expression fragment in the suicide vector, pSUP202. Successful exconjugants were selected as $\mathrm{Ps}^{+} \mathrm{Km}^{\text {" }} \mathrm{Tc}^{\text {". }}$ Maintenance of the mutation even after photohcterotrophic growth was established by dot blot analysis. This is consistent with the very similar growth rates of the M234 mutants compared to the deletion strain complemented with wild-type reaction-center genes.

The four mutations at position 234 of the M-subunit are:

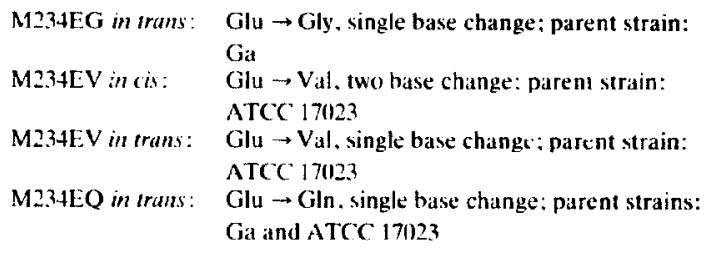

Bacterial culture. Rb. sphaeroides cells were grown in Sistrom's minimal medium [18] supplemented with 0.2\% casamino acids. Kanamycin $(25 \mu \mathrm{g} / \mathrm{ml})$ and tetracycline $(2 \mu \mathrm{g} / \mathrm{ml})$ were present for the in trans mutants. $50 \mathrm{ml}$ cultures were grown aerobically, in the dark. by vigorous shaking at $30^{\circ} \mathrm{C}$, and then inoculated into 2 litres of medium for continued growth under semi-aerobic conditions to induce pigmentation and synthesis of photosynthetic components, including reaction centers. In some cases, the semiaerobic cultures were inoculated into 9.5 litres of Sistrom's medium, and grown under light for 4 days before harvesting. Sequencing of the M-subunit gene in plasmids isolated from light grown cells showed that there was no primary site reversion or any secondary site mutations within 100 bases of the M234 codon.

Chromatophore and reaction center preparation. Chromatophores, for in situ optical measurements, were prepared from $R b$. sphaeroides strain Ga, and 
from the various mutants, as previously described [19]. Reaction centers were prepared from $R b$. sphacroides strain ATCC 17023, strain Ga and the various mutants derived from these, by detergent fractionation of chromatophores with lauryldimethylamine $N$-oxide (LDAO). RCs were further purificd by $\left(\mathrm{NH}_{4}\right)_{2} \mathrm{SO}_{4}$ precipitation and by DEAE-Sephacel ion exchange chromatography $[19,20]$. Secondary acceptor activity was normally reduced to about $0.3-0.5 \mathrm{Q}_{B}$ per RC. When desired, supplemental ubiquinone $(20 \mu \mathrm{M})$ was added from a $20 \mathrm{mM}$ stock suspended in $30 \%$ Triton $\mathrm{X}-100$.

Optical measurements. Kinetic absorbance measurement in the microseconds to seconds time range were performed on an unchopped spectrophotometer of local design [20], with excitation provided by a xenon flash lamp ( $8 \mu \mathrm{s}$ fwhh). Measurements of picosecond kinetics were performed on the apparatus described by Holzapfel et al. [21]. The excitation pulse width was less than $200 \mathrm{fs}$.

EPR measurements. Low-temperature EPR spectra were obtained using a Bruker 200D EPR spectrometer equipped with an Oxford Instruments Liquid Helium dewar system. Samples $(0.3 \mathrm{ml}$ of approx. $30 \mu \mathrm{M}$ RCs $)$ were in $3 \mathrm{~mm}$ i.d. quartz tubes and were chemically reduced by the addition of $5 \mathrm{mM}$ srdium dithionite immediately hefore freezing. Deriatiw spectra were obtained under field modulation $(10 \mathrm{HHz})$ conditions.

Formate-bicarbonate treatment. Bicarbonate was depleted from the ciromatophores and reaction center samples essentially as previously described [22]. Sodium formate was added to the sample to $1010 \mathrm{mM}$ at $\mathrm{pH} 6.0$ and the sample degassed and flushed with $\mathrm{CO}_{2}$-free $\mathrm{N}_{2}$, prepared by passing $\mathrm{N}_{2}$ gas through a column of $\mathrm{CaCl}_{2}$ and ascarite before rehydrating it with distilled water. The bicarbonate depletion procedure was conducted at $25^{\circ} \mathrm{C}$ for at least $2 \mathrm{~h}$ to ensure complete depletion.

\section{Results and Discussion}

Charge recombination of $P^{+} Q_{-4}^{-}$and $P^{+} Q_{B}^{-}$

Following a flash, in the absence of secondary donors, the decay of $\mathrm{P}^{+}$in isolated $\mathrm{RCs}$ reflects recornbination of the charge separated states. $P^{-} Q_{A}^{-}$and $\mathrm{P}^{+} \mathrm{Q}_{A} \mathrm{Q}_{\mathrm{B}}^{-}$, depending on the reconstitution of $\mathrm{Q}_{\mathrm{B}}$ function by the addition of ubiquinone. The decay of $\mathrm{P}^{+}$in the presence of ubiquinone occurs by recombination of
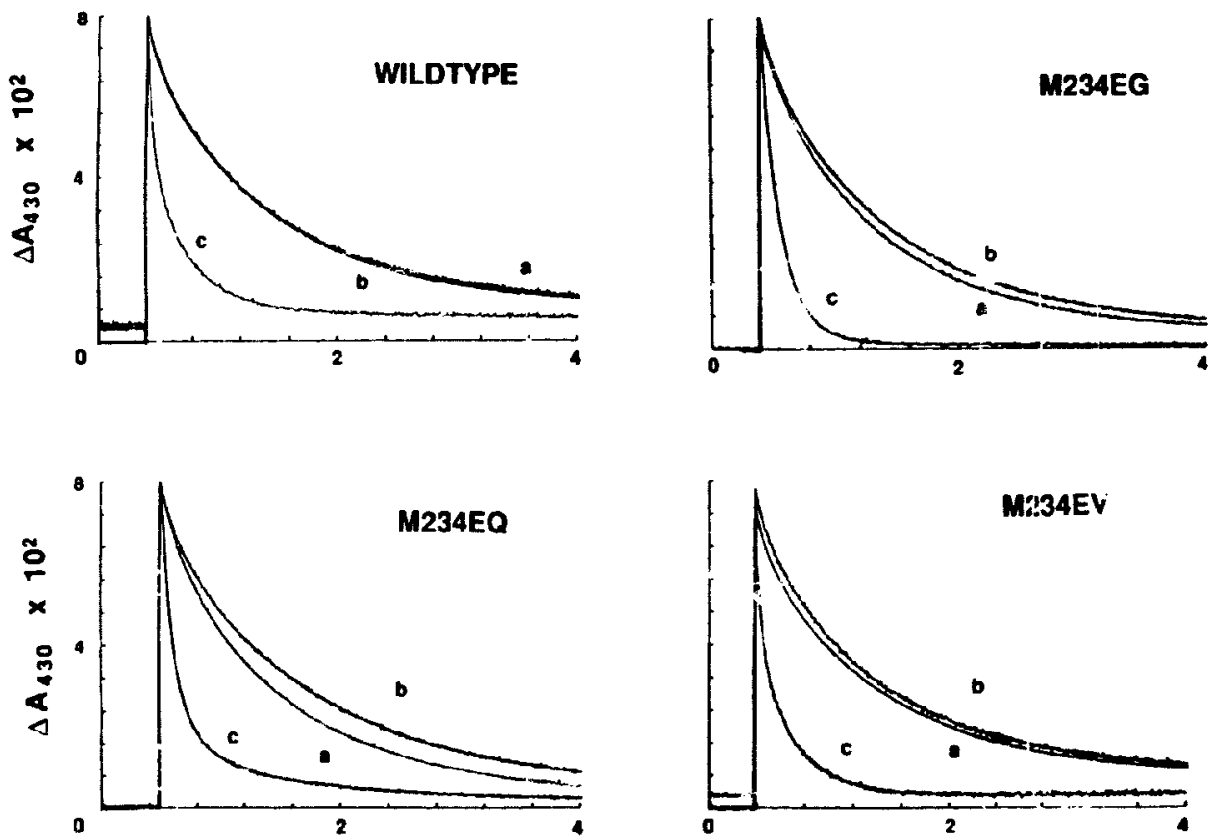

TIME (s)

Fig. $1 . P^{+} O_{i}$ and $P^{+} O_{13}$ recombination kinetics in $W t$ and $M 234$ mutant reaction centers. Recovery of the oxidized primary dimor in the ahsence of exogenous donor, measured at $430 \mathrm{~nm}$. Conditions: $2 \mu \mathrm{MRCs}$ in 116$) \mathrm{mM} \mathrm{KCl}$ and $10 \mathrm{mM}$ Bistris at pH $6.020 \mu \mathrm{M}$ uhiquinone- 10 was added with 0.039; Triton X-100. (a) No further additions: (b) bicarbonate depleted with ! (6) miM sodium formate and degassing a! pll f.0: (c) with 6) $\mu \mathrm{M}$ terbutryn. Wild type $\left(W_{1}\right)$ and mutants $\mathrm{M} 234 \mathrm{EG}$. M234EO and M234EV an indicated. 
$P^{-} Q_{1} Q_{13}$ through equilibrimm with the $P^{*} Q_{\text {, tate as }}$ follows [23.24]:

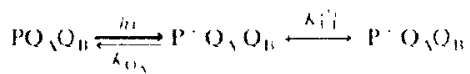

$K_{1}^{\prime \prime}$ is the equilibrium constant for the one electron transfer between $Q_{A}$ and $Q_{k}$ and $k_{O}$ is the ratce of recombination of $P \cdot Q_{A}$. Since the electron-transtier cyuilibriun is rapid compared to $k_{0}$, the ralle constant for recombination of the $\mathrm{P}^{+} \mathrm{O}_{B}$ state is given by $[2.3-25]$ :

$A_{O_{H}}=h_{U_{1}}\left[1+K_{1}^{\prime \prime \prime}\right]^{\prime}$

(Binding of quinone to the $Q_{4}$ site is presumed saturated.) Thus, measurement of the rales of recombination of $\mathrm{P}^{+} \mathrm{Q}_{1}\left(k_{0,}\right.$. determined in the absence of functional $\left.Q_{13}\right)$ and $P^{+} Q_{13}\left(k_{Q_{4}}\right.$ determined in the presence of excess quinonc) allows calculation of $K_{1}^{(11}$.

Medsurement of the charre recombination kinctics in isolated RCs showed very similar kinetics for the fast $P^{\prime} O_{A}$ recombination or the som $P^{\prime} O_{B}$ back-reaction rate in $W_{1}$ and M23t mutars $P C s$ at $\mathrm{pH} 6.0$ (Fig. 1), and also at $\mathrm{pH} 8.0$ anc 10.0 (not snown). Furthermore. formatc had no significant effect on these kinctics in any of the RC preparations (compare traces a and b). Bicarbonate (1!) $\mathrm{mM}$ ) itself had no effect (data not shown). Thus, neither the mutational changes nor formate at fected the equiliorium sharing of an electron between the : wo quinones.

Even in the presence of added ubiquinons, a small amplitude of the last phase of $\mathrm{P}^{+}$decay is seen in isolated RCs; this reflects $R C_{\text {s }}$ lacking $Q_{1}$, either because of incomplete saturation of the $Q_{13}$-binding site or because of damage leading to non-reconstitutibility [24.25]. The fraction of fast phase was similar in all preparations, ranging from $12 \%$ in $\mathrm{Wt}$ and $\mathrm{M} 234 \mathrm{EV}$ mutant RCs to $20 \%$ in $\mathrm{M} 234 \mathrm{EG}$ and, again, formate wats without effect. Since $20 \mu M \mathrm{Q}-10$ is sufficient to saturate the $Q_{13}$-binding site in Wt RCs [26\%. this fraction largely reflects non-reconstitutihle RCs. Terbutryn. a comp ". ive inhlitor of $Q_{13}$ activity. strongly suppressed the : low phatse of the back reaction in all preparations see curves $c$ in Fig. 11. Thus, the mutations did not markedle wifect the binding or functional proper ies of the quinone sites.
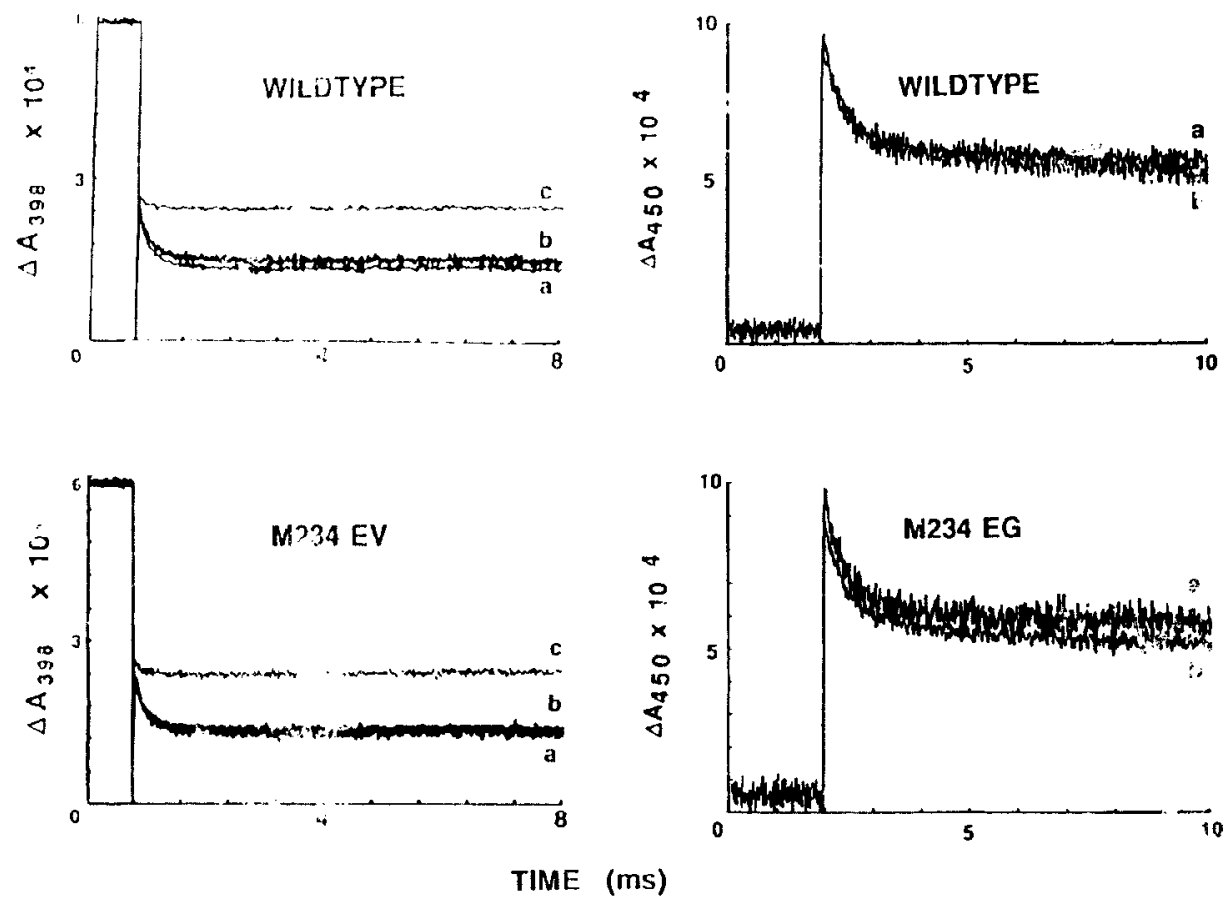

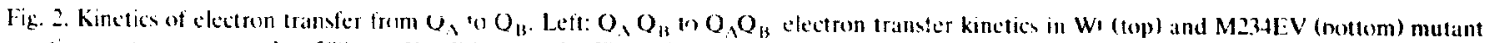
reaction cenics, measured at $398 \mathrm{~mm}$. Conditosis: as for Fig. 1. (a) No further addition: (h bicarboate deplefed with $100 \mathrm{mM}$ sodiem formate

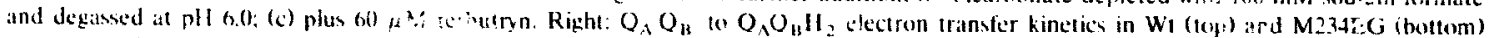

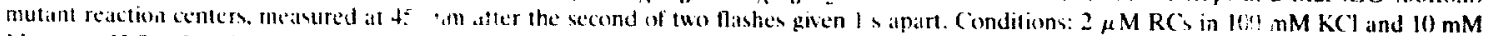

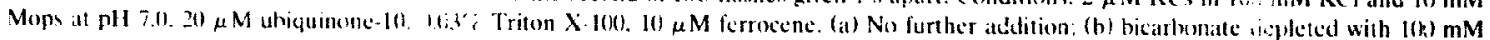
sodium formate and degassed at pH 6.0. 
Qirect measurement of electron transfer from $Q_{+}$io $Q_{B}$ and from $Q_{\text {i }}$ to $Q_{B}$

Transfer of the first electron from $Q_{A}$ to $Q_{H}$. folbising a single hash. Was detected by measuring the aid orbance change at $398 \mathrm{~nm}$. The kinctics for $W t$ and 1.234EV mutant RCs. at pH 6.l. are shown in Fig. 2 : $f$ ft panels): identical results ware obtained for M?34EC and M234EQ rutant RCs. The instantasoous absorption change is duc to $P^{+} Q_{A}$. In Wt RCs, the subsequent electron transfer from $Q_{i}$ to $Q_{B}$ occl:rred with a half time of about $150-170 \mu \mathrm{s}$ when analyzed as a single exronential component. In all the :234 nutant RCS, the half-time was similar (170-180 $\mu s$. Treatment of $\mathrm{Wt}$ or mutant RCs with $100 \mathrm{mM}$ formatc and flushing with $\mathrm{N}_{2}$ for about $2 \mathrm{~h}$ at $\mathrm{pH} 6.0$ iid not change the kinetics of electron transfer from $O_{\text {、 }}$ to $Q_{\mathrm{H}}$ (compare traces a and b). The inhibitor i. rhutryn effectively blocked electron transfer in all a preparations (see curves c). Bicarbonate (10 $\mathrm{mM}$ ) had no effect (data not shown). The $W t$ and all three M234 mutant RCs also exhibitcd similar electron transfer kinctic: at $\mathrm{pH} 8.0$ (not shown).

The kinetics of electron transfer from $Q_{A}^{-}$to $Q_{B}$ fter the second flash. were determined in the presnce of a low concentration of ferrocene, sufficient to irap $P Q, Q_{13}$ sifter the first flash. Following a second Fash. the elect on transfer from $Q_{A}$ to $Q_{B}^{-}$was seen as a rapid sisappearance of the semicuinone absorbanee signal at $450 \mathrm{~nm}$ (Fig. 2, right pands), preceding the decay of $P$ due 10 slow electron donation from ferrecene. For Wt RCs. at $\mathrm{pH} 7$, the half time for clectron transfer was 270 $\mu \mathrm{s}$. In M234EG and M234EV mutant RCs, the halt-time was $330 \mu$ s. Formate treatment had no consistent eifect $(\leq 15 \%$; change) on any preparation. and bicarbonate (11) $\mathrm{mM}$ ) had no effect (not shown).

It is evident from these results that neither the first nor the second electron transfer to $Q_{13}$ was kinetically impaired, or even detectably altered, in the M234 mutant RCs.

\section{Two-electron gate oscillations of semiquinone formation and disappearance'}

In PS II and the purple bacteria, export of reducing cquivalents is achieved through the acceptor quinone complex where the second quinone $\left(Q_{B}\right)$ functions as a two-clectron gith . . wsing reducing equivalents out of the RC only in patts [27].
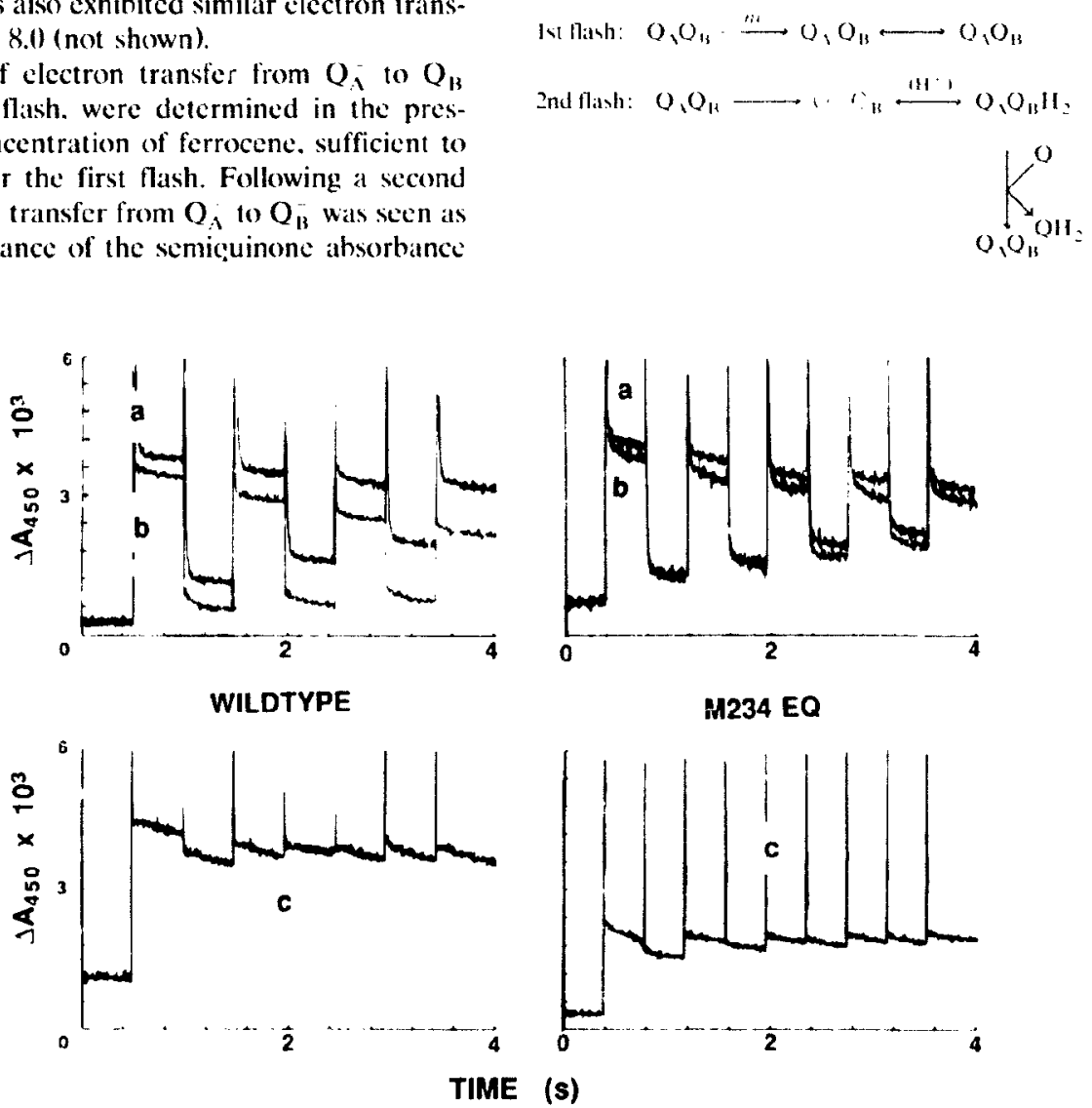

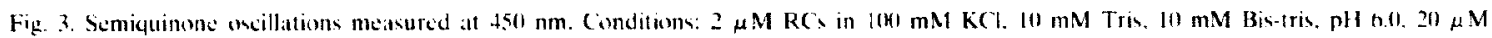

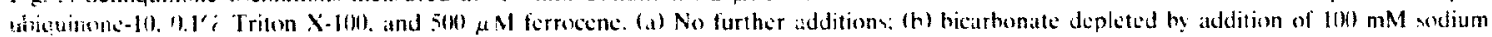

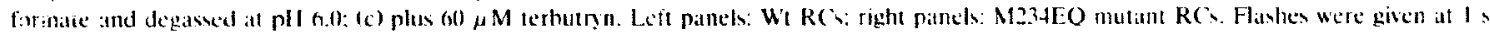
intervals. 
$\left(\mathbf{P}^{+}\right.$is re-reduced after each flash and is omitted from this scheme.) $Q$ represents the quinone pool of the electron transport chain. Binary oscillations in the formation and disappearance of the stable anionic semiquinone of $Q_{B}$ are shown in Fig. 3 (top panels). A stable semiquinone signal is observed after an odd number of flashes, giving an oscillation with a period of two (measured at $450 \mathrm{~nm}$ ). The electron transfer equilibria heiween $Q_{\text {, and }} Q_{B}$ generate a small population of $Q_{A}$, in which state the RC fails to turnover upon subsequent activation. This leads to damping of the oscillations [23,28]. Very similar oscillatory patterns were seen for $W t$ and M234EQ nutant $R C s$, and formate treatment did not affect the oscillation of the semiquinone signal in either type. The differences between traces $a$ and $b$ are duc to some dilution during the formate treatment. and to baseline drifts due to differences in light seattering of the two samples. They do not reflect a significant change in the functional parameters of the oscillatory activity. This result indicates that the mutational change at M234 did not alter either the first or the second electron transfer equilibrium between $Q_{A}$ and $Q_{B}$, or the yield of quinone reduction. Oscillations in the semiquinone signal were abolished by 6) $\mu \mathrm{M}$ terbutryn in all samples (Fig. 3, bottom panels).

\section{Electron flow in chromatophores}

In $R b$. sphacroides, light-induced charge separation in reaction centers is followed, in situ, by a cyclic electron transfer from $\mathrm{O}_{\mathrm{B}}^{-}$back to $\mathrm{P}^{+}$via the whiquinone pool and cytochrome $b / c$, complex in the membrane, and cytochrome $c$, in the periplasm. Electron transfer to the $b / c_{1}$ complex was determined in Wt and M234EV mulant chromatophores by following the reduction of cytochrome $b-561$. measured from the absorbance difference between 561 and $574 \mathrm{~nm}$ (Fig. 4). Valinomycin was present to eliminate clectrochromic shifts associated with the membrane electrical potential, and antimycin $\mathrm{A}$ was added to block the rapid reoxidation of $b$-type cytochromes. Ferricyanide was added in an attempt to fully oxidize $Q_{13}$, but this component is notoriously difficult to equilibrate [29]. The fact that cytochrome $b$ reduction was observed after a single flash indicates that $Q_{B}$ was not equilibrated with the prevailing redox potential. but was present in a roughly 50:50 mixture of $Q_{13}$ and $Q_{13}$, essentially the steady-state condition. The extent and kinetics of reduction of cylochrome $b-561$ were the same in both Wt and M234EV mutant samples, and showed no sensitivity to formate treatment or to addition of bicarbonate $(10 \mathrm{mM}$. not shown) in either $\mathrm{Wt}$ or mutant chromatophores. However. terbutryn was inhibitory, as expected (not shown). At the high ambient redox potentials used hure, the kinetics of cytochrome $b-561$ reduction are very slow and non-exponential due
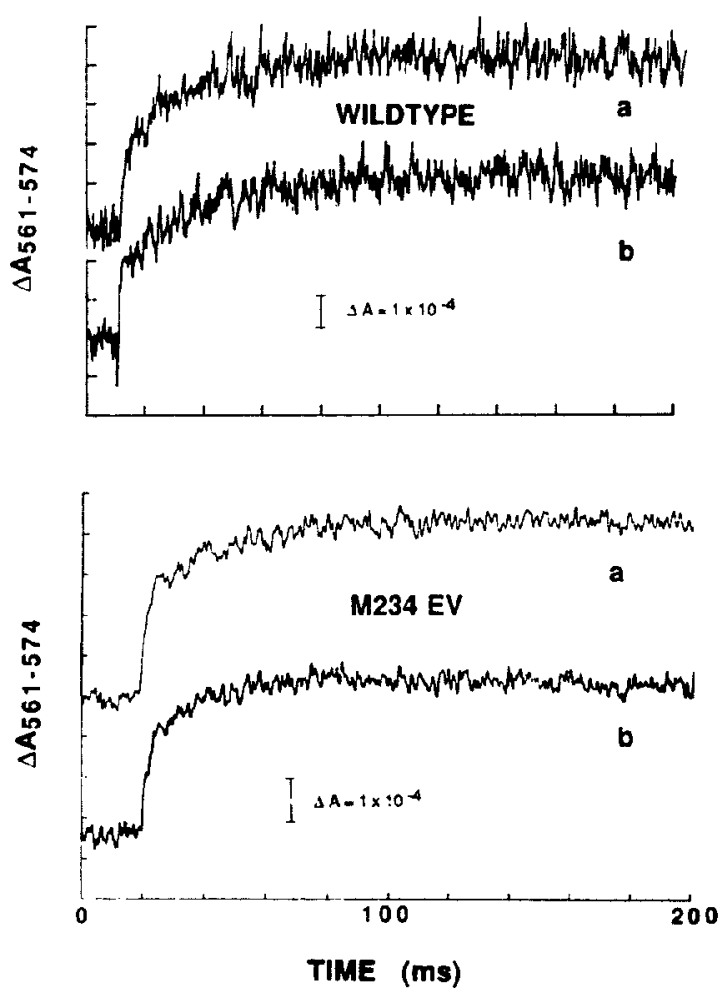

Fig. 4. Reduction of cytochrome $\mathrm{b}_{56]}$ in chromatophores, measured hy the absorbance change at 561 -minus- $574 \mathrm{~nm}$. Chromatophores were added to $100 \mathrm{mM} \mathrm{KCl}$. $10 \mathrm{mM}$ Tris and $10 \mathrm{mM}$ Bistris (pl 0.0 ) to an absorbance of 2 at 800$) \mathrm{nm}$. Ferricyanide $(\approx 100 \mu \mathrm{M})$ was added to raise the redox potential to 380$)-400 \mathrm{mV}$, and $2 \mu \mathrm{g} / \mathrm{ml}$ valinomycin and $2 \mu \mathrm{g} / \mathrm{ml}$ antimycin $\mathrm{A}$ were also present. (a) No further addition: (b) bicarbonate depleted with addition of 109$) \mathrm{mM}$ sodium formate, and degassed at $\mathrm{pH} 6$. Top panel: Wt chromatophores: bottom panel: M234EV mutant chromatophores.

to the second-order nature of the reaction $[30,31]$. The process involves the reduction and release of quinol from the reaction centers and diffusion of quinol to the cytochrome $b / c_{1}$ complex. with an overall halftime of $10-20 \mathrm{~ms}$. The essential identity of the $\mathrm{W}_{\mathrm{t}}$ and mutant kinetics strongly indicates that all potentially rate-limiting steps are unaltered in the mutants.

\section{LPR signals of the $Q_{A}$ semiquinone}

The electron acceptor quinone complex of bacterial and PS II RCs is centered on an iron atom. Although the iron probably does not play an active redox role in electron transfer, it does exchange couple with the semiquinone species of $Q_{A}$ and $Q_{B}$ to give rise to unusual and very distinctive low-temperature EPR signals for $\mathrm{Q}_{\mathrm{A}}^{-}-\mathrm{Fe}^{11}$ and $\mathrm{Q}_{\mathrm{B}}^{-}-\mathrm{Fe}^{\prime \prime}$, centered at $\mathrm{g}=1.82$ [32.33]. It is reasonable to expect these signals to be sensitive to the structural integrity of the acceptor quinone complex. The low temperature EPR signals 


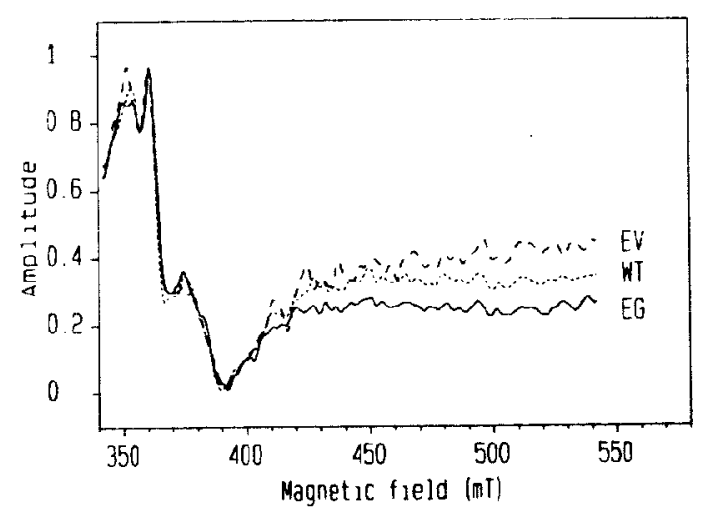

Fig. 5. Low-temperature EPR spectra of $Q_{A}-F{ }^{11}$ in $W_{1}$ and $M_{2.34}$ mutant RCs. Samples containing 31 $\mu \mathrm{M}$ RCs. in I(K) $\mathrm{mM} \mathrm{KCl.} 10$ $\mathrm{mM}$ Tris (pII \&.(0), nere reduced with $5 \mathrm{mM}$ dithicnit. immedialely before freczing. Spectrometer scttings: microwave pows $=20 \mathrm{~mW}$ : frequency $=9.300 \pm 0.002$ GHz: nodulation amplitude $=20 \mathrm{G}: \mathrm{lcm}$. perature $=5 \mathrm{~K}$. Dotted line: Wt RCs; dashed line: M234EV mutan! RCs: solid line: M234EG mutant RCs.

for $\mathrm{Q}_{\mathrm{A}}^{-}-\mathrm{Fe} \mathrm{e}^{11}$ in Wt, M234EV and M234EG RCs, reduced by dithionite at $\mathrm{pH}$ 8.0, are shown in Fig. 5 . Similar results were obtained at $\mathrm{pH} 6.0$ and 10.0 . There is no appreciable difference between $W_{t}$ and the $\mathbf{M} 234$ mutant RCs. implying a remarkable conservation of the coupling and structural parameters of the interaction.

\section{Primany ecents}

The non-essentiality of the iron atom in acceptor quinone function has been demonstrated by the elegant extraction and reconstitution experiments of Debus et al. [34]. Removal of the iron, with careful retention of the H-subunit, caused only a 2-fold slowing of the first electron transfer to $Q_{B}$ and a 3.4 fold slower steady-state turnover rate. The latter includes a significant decrease in the quantum yicld of $Q_{1}$ reduction which later measurements showed to be due to an unexpectedly large effect on the rate of reduction of $\mathrm{Q}$, by $\mathrm{BPh}^{-}$, which was slowed from $200 \mathrm{ps}$ to $4 \mathrm{~ns}$ [35]. Sub-nanosecond measurements on M234 mutant RCs showed no effect of the mutations on the very early events leading to reduction of bacteriopheo- phytin. $H_{A}$, which have recently been resolved into two phases reflecting reduction of the atcessory bacteriochlorophyll. $\mathrm{B}_{\mathrm{a}}$, in $3.5 \mathrm{ps}$, followed by electron transfer to $\mathrm{H}_{1}$. with a chatacteristic time of $0.9 \mathrm{ps}[21]$. How. ever, the subsequent clectron transfer to $Q$, was 2-fold slower in M234EQ and M234EV mutant RCs than in Wt RCs (Table I).

Replacing glutamate with glutamine, glveinc or valine must eliminate one or more of the native ligands contributed from Gilatist to the iron. as well as the partial compensation of the ferrous ion charge provided by an ionized glutamete. It would seem reasonable to expect, therefore. possibly significant structural alterations in the mutants - either to obtain suitable charge compensation and liganding from other nearby candidates (there are several acidic groups in this region of the structure) or to eliminate the iron atom altogether. However. the EPR signals of the $Q_{A}$ semiquinone in $W t$ and mutant $R C s$ indicate that any perturbations of the acceptor quinone complex do not affect the exchange coupling through the intervening histidine ligands. This result is partially confounded by the mild, but definite. effect of the mutations on the picosecond kinetics of electron transfer from $\mathrm{BPh}^{-}$to $Q_{A}$. It is difficult to see, in a simple model, how this can be compatible with no perturbation of the $Q_{1}-F$ "I EPR signal, which should be a rather sensitive probe of both electrostatic and structural influences [3.3]. In PS 11. for example. formate-induced depletion of bicarbonate causes a marked narrowing and intensification of this signal, although it is not affected by some herbicide resistance mutations in the $Q_{13}$ pocket [36].

\section{Conclusions}

Studies on the bicarbonate effect in oxygen-evolving organisms have suggested two roles, possibly associated with two distinct binding sites $[1-4.10]$ : (1) as a direct ligand with Fe"l. to provide a stable conformation for functional electron transfer; (2) as a proton donor to the plastoquinone reductise site in the DI-D2 protein. The kinetic behavior of Wt and Glu ${ }^{234}$ mutant RCs of Rh. sphacroiles is summatrized in Table I. which lists the lifetimes for all electron transfer steps involved in

TABLE 1

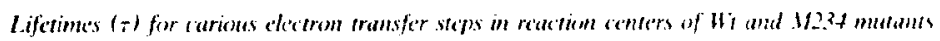

\begin{tabular}{|c|c|c|c|c|c|c|c|}
\hline & \multicolumn{2}{|c|}{$\mathrm{P}^{+} \mathrm{O}_{\text {recombination }} \quad \mathrm{P}^{+} \mathrm{Q}_{\mathrm{B}}$} & \multirow[t]{2}{*}{$\underset{(\mu N)}{O_{B}} \cdots O_{B}$} & \multirow[t]{2}{*}{$\begin{array}{l}Q_{A} \rightarrow Q_{3} \\
(\mu s)\end{array}$} & \multirow[t]{2}{*}{$\begin{array}{l}p+\ldots B_{1} \\
(p)\end{array}$} & \multirow[t]{2}{*}{$\begin{array}{l}\mathrm{B}_{1} \rightarrow \mathrm{H}_{1} \\
(\mathrm{p}, \mathrm{l}\end{array}$} & \multirow[t]{2}{*}{$\begin{array}{l}\mathrm{H}_{1} \rightarrow \mathrm{O}_{1} \\
(\mathrm{ps})\end{array}$} \\
\hline & $\mathrm{ms}$ & $\mathrm{ms}$ & & & & & \\
\hline$\overline{W_{1}}$ & $170 \pm 15$ & $1125+30$ & $225+20$ & $39(1+31)$ & $3.5+0.4$ & {$[1.4+0.3$} & $22(1+20$ \\
\hline $\mathrm{M} 234 \mathrm{EQ}$ & $165 \pm 15$ & $1095+30$ & $200+20$ & - & $3.5+0.4$ & $0.9+0.3$ & $411+211$ \\
\hline M234EV & $155+15$ & $10901 \pm 30$ & $24.5 \pm 211$ & $4(0,1)+411$ & $35+11.4$ & $11.9+11.3$ & $471+20$ \\
\hline M234EG & $16.5 \pm 15$ & $1170+30$ & $245+20$ & $440+411$ & - & .. & - \\
\hline
\end{tabular}


the turnover of the reaction center. The mutants showed almost wild-type behavior for all processes and cxhibited no effect of hicarbonate-depletion (formate treatment) on electron transfer from $Q_{i}$ to $Q_{13}$ and from $Q_{A}$ to $Q_{B}$. oll the two clectron gate operation. or on the decay of the $P^{+} Q_{A}$ and $P^{+} Q_{B}$ states. Experiments on chromatophores from wild-type $R h$. sphatroides (Strain Ga) and from mutant M234EV showed no significant formatc/bicarbonate effect on the light-initiated electron transfer from $Q_{\mathrm{A}}$ to $\mathrm{cy}$ tochrome b-561. Furthermore, the EPR signal of the $\mathrm{Q}_{1}-\mathrm{Fe}^{11}$ spin system suggests that any perturbations that may accompany the mutational substitutions at M234 are very small. These results inply that the structural integrity and function of the acceptor quinone complex in bacterial RCs, including the central iron-histidine ligand interaction. is not very sensitive to the provision of the fifth and sixth ligands by Glu ${ }^{N 2: 4}$. It seems, therefore. that the requirement for bicarbonate in PS 11 . is not accounted for by a simple bicarbonate / carboxylate Fe-ligand homology, although it must be recognized that there are other primary structure differences between PS II and bacterial RCs. in this region. A possible involvement in proton transfer is not addressed directly as Glu ${ }^{\text {M234 }}$ is not implicated in such an activity in hacterial RCs. However, recent site-directed mutagenesis studies on other carboxylic residues in the $Q_{B}$ binding site of $R h$. sphacroides RCs have revealed a crucial role for Glu1.212 and Asp ${ }^{1213}$ in proton transfer activities, and mutational lesions at these sites exhibited hehavior reminiscent of hicarbonate-depleted activites in PS 11 [37]. The relationship between these observations is under further study.

\section{Acknowledgements}

This work was supported by a grant from the Unitcd States Department of Agriculture, Competitive Rescarch Grants Office to C.A.W. (USDA/CRGO $A G 89-37262-4462)$, and by the Deutsche Forschungsgemeinschaft (SFB 143). J.C. gratefully acknowledges receipt of a traineship from the Mcknight Foundation.

\section{References}

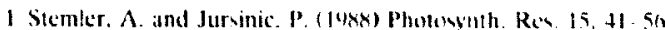

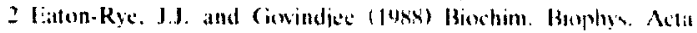
9.35, $237 \ldots 247$

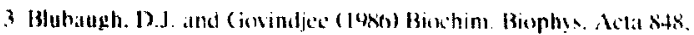
$1+7-1.1$.
I Bluhaugh. D.J. and (Jovindjee (1988) Photosynth. Res. 19, 85-128.

5 Shopes. R.. Bluhatugh. D.J.. Wraight. C.A., and Govindjec (1989) Biochim. Biophys. Acta 947. 111-11\%.

h Datsenhofer, J., Epp. O.. Miki, K. Jubir. R. and Michel. H. (14sit) J. Mol. Biol. I $80,385-398$.

7 Nlen, J.P. Feher, G. Yeates, T.O. Komiya, H. and Rees. D.C. (1057) Prix. Natl. Aciad. Sci. USA 84, 5730)-5734.

8 Allen. J.P. Feber. G. Yeates. T.O. Komiya, H. and Rees. D.C. (1958) Proc. Natl. Acad. Sci. US A 85, 8487-.8441.

9 Michel. H. and Deisenhoter. J. (1988) Biochemistry 27. 1-7.

10 Dincr. B.A.. Petrouleas, V. and Wendolski. J.J. (1991) Physiol. Plint si. $423-4.36$.

1) Farchaus, J.W. and Oesterhelt, D. (1989) EMBO J. 8. 47-54.

12 Takahashi. E.. Maróti. P. and Wraight, C.A. (1989) in Current Topics in Photosynthesis. Vol. I (Baltscheffsky, M., ed.), pp. 169-172, Kluwer. Dordrech!.

13 Willams. I.C.. Stainer. L.A., Ogden, R.C. Simon, M.I. and Feher, G. (198.3) Proc. Natl. Acad. Sci. USA 80, 6505-6509).

14 Simon. R., Preifer. U. and Puhlet. A. (1983) Biotechmolugy 1. $7 \mathrm{sit}-791$

15 Kunkcl. T.A. (1945) Proc. Natl. Acad. Sci USA. 82, 488-492.

16 Maniatis, T., Fritsch. E.F. and Sambrok, J. (1982) Molecular Claning. Cold Spring Harbor Laboratory. Cold Spring Harbor.

17 Davis, J., Donohues. T.J. and Kaplan, S. (1988) J. Bacteriol. 170. $32(1-324)$.

I8 Sistrom. W.R. (1W601) J. Cien. Microbiol. 23. 778-785.

19 Maróli. P. and Wratight. C.A. (1488) Biochim. Bophys. Acta 9.34. $314-328$.

2) Stcin. R.R. (1985) Ph.D.thesis, University of Illinois. Urbana.

21 Ilokapfol, W., Finkels. U., Kaiser. W.. Oesterhelt. D., Scheer, H., Stilz. H.U. and Zinth, W. (1989) (hem. Phys. Let1. 160. I-7.

22 Blubaugh. D.J. and Govindjec (1988) Biochim. Biophys. Acta 936. 2 (IS- 214 .

23 Wraight. (. A. (1979) Biochim. Biophys. Acta 548, 3119-327.

24 Kleinfeld. D. Ohamura. M.Y. and Feher, G. (1984) Biochim. Biophys. Actia $760.126-141$.

25 Stein. R.R., Castellvi, A.L., Bugacz. J.P. and Wraight, C.A. (1984) J. Coll Biochem. 24. 27.3-259.

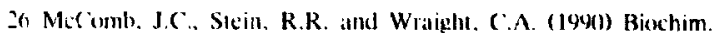
Buphys. Acta IIIIS, 150,171.

27 Wraight, ( . A. (1982) in Function of quimones in encray conserving systemu ( Trumpower, 13.1.. cd.). pp. 181-198. Academic Press. New York.

28 Kleinfeld. D., Ahresch. E. C.. Okamura, M.Y. and Feher, G. (1984) Buchim. Biophys. Acla 765, 406-404)

20) ()keste. D.P. Prince. R.C. and Dutton. P.L (1981) Binchim. B1tophys, Acta $6.37,512-522$.

30) Matsulura. K. O'Kerfe, D.P. and Duton. P.L.. (1983) Binchim. Bioplysis. Acta 722, 12-22.

31 (rofts, A.R., Meinhardt. S.W.. Jonss, K.R. and Snozzi. M. (1983) Biochim. Biphys, Acta 723, 2(02-218.

32 Wratight. (.A. (1978) FEBS 1.211, 93, 28,3-288.

3 Butler. W.F., Cituo, R. Fredkin. M.R. Isaticonn, R.A. Okamura, M. Y. and Foher. (i. (1984) Biophys. $1.45,9+7.973$

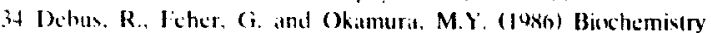
25. $2276.22 \times 7$.

35 Kirmaici, (.. Hollen, D., Debus, R.J.. Feher. G. and Okamura, M.Y. (l966) Proc. Natl. Acad. Sci. USA 83, (24107-6411.

36 Vermass. W.F.I and Rutherford, A.W. (1964) FEBS Lett. 175. $2+3-2.48$.

37 Takahashi. E. and Wraight, C.A. (1491) FEBS Lett. 283, 140-144. 